\title{
Granulocyte Colony-Stimulating Factor-Producing Carcinoma of Unknown Primary Site
}

\author{
Hirotoshi Yasui ${ }^{a}$ Kazuhide Sato ${ }^{a}$ Yoshihiro Takeyama ${ }^{a}$ Akira Ando ${ }^{a}$ \\ Toshio Kato $^{a}$ Hiroyuki Hashimoto $^{a}$ Yasutaka Fukui $^{a}$ \\ Matsuyoshi Maeda ${ }^{b}$ Hideo Gonda ${ }^{a}$ Ryujiro Suzuki ${ }^{a}$ \\ Departments of ${ }^{a}$ Respiratory Medicine and ${ }^{b}$ Clinical Pathology, Toyohashi Municipal \\ Hospital, Toyohashi, Japan
}

\section{Key Words}

Carcinoma of unknown primary site $\cdot$ Granulocyte colony-stimulating factor-producing tumor $\cdot$ Carboplatin + paclitaxel + bevacizumab + erlotinib

\begin{abstract}
Granulocyte colony-stimulating factor (G-CSF)-producing nonhematopoietic malignancies have been reported in various organs and are associated with a poor clinical outcome. Moreover, carcinoma of unknown primary site (CUP) is an uncommon malignancy that occurs in about $2-6 \%$ of cancer patients. CUP also has a poor prognosis due to its missing profile. Since both G-CSF-producing carcinoma and CUP are rare, G-CSF-producing CUP (GCSF-CUP) is considered to have an even poorer prognosis and is seldom encountered. Herein, we report the case of a GCSF-CUP patient. A 75-year-old man was admitted to our hospital complaining of cervical lymphadenopathy. Multiple bulky lymph nodes without a primary site were revealed by image analysis. His complete blood count showed leukocytosis, and his blood chemistry panel indicated highly elevated levels of G-CSF. Although the patient was treated with combination chemotherapy of carboplatin, paclitaxel, bevacizumab and erlotinib, he died of intestinal perforation due to tumor invasion 23 days after the start of the therapy. An autopsy confirmed that the tumor was positive for anti-G-CSF antibody, but the primary site was still not detected.


Yasui et al.: Granulocyte Colony-Stimulating Factor-Producing Carcinoma of Unknown Primary Site

\section{Introduction}

Malignancy-producing granulocyte colony-stimulating factor (G-CSF) is rare. Robinson [1] first described a G-CSF-producing tumor (GCSF-PT) in 1974, and it has since been reported in various sites of cancer. G-CSF is a glycoprotein that stimulates the proliferation and maturation of precursor cells in the bone marrow into fully differentiated neutrophils $[2$, 3]. G-CSF produced by tumor cells acts on itself, progressing rapidly [4]. Therefore, GCSF-PT generally has a poor prognosis.

Carcinoma of unknown primary site (CUP) is also a rare tumor, accounting for approximately $3 \%$ of all cancer diagnoses. CUP often has a poor outcome due to its early dissemination, aggressive behavior and unpredictable nature as well as its metastatic potential and pattern [5]. Although platinum/taxane combination (e.g., CBDCA + PTX) chemotherapy is widely used for patients suffering from CUP, its response rate is only about $30-40 \%$, with a median overall survival of merely 9 months. Among new regimens, the combination of carboplatin, paclitaxel, bevacizumab and erlotinib is thought to be highly desirable [6].

So far, CUP which produces G-CSF (GCSF-CUP) is too uncommon to be treated with a precise chemotherapy regimen. We herein report a challenging case of GCSF-CUP with aggressive growth, treated with combination chemotherapy of carboplatin, paclitaxel, bevacizumab and erlotinib.

\section{Case Presentation}

A 75-year-old man presented to his primary care clinic due to a left neck lump and was referred to our hospital. He had broken his left ankle in a traffic accident, and he had been smoking half a pack of cigarettes per day for 55 years. Some lymph nodes were palpable on the left side of the neck (fig. 1a). His complete blood count and blood chemistry were as

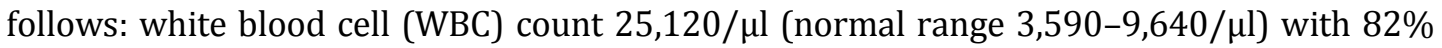
neutrophils, $3 \%$ monocytes and $8 \%$ lymphocytes; no immature granulocytes were observed; platelet count $21.8 \times 10^{4} / \mu \mathrm{l}$ (normal range 14.8-33.9 $\times 10^{4} / \mu \mathrm{l}$ ), and C-reactive protein level $4.45 \mathrm{mg} / \mathrm{dl}$ (normal level $<0.3 \mathrm{mg} / \mathrm{dl}$ ). Various tumor markers were elevated, and SCC, CYFRA and CA19-9 were extremely increased (table 1). His WBC count increased gradually up to $44,680 / \mu \mathrm{l}$, with a differential count of $94 \%$ neutrophils during a 6-week period after his first visit, while the serum G-CSF concentration (normal range $3.7-32.3 \mathrm{pg} / \mathrm{ml}$ ) was increased as high as $283 \mathrm{pg} / \mathrm{ml}$ during the same period, suggesting that the tumor grew rapidly.

A contrast-enhanced CT scan showed massive lymphadenopathy of the left cervix, left subclavian artery, left mediastinum, mesenteric and para-aortic lymph nodes, with peripheral rim enhancement, consolidation in the right lower lung and a 1-cm low-density liver lesion (fig. 2a-d). ${ }^{18} \mathrm{~F}-\mathrm{FDG}$ PET/CT demonstrated a high FDG accumulation in the lymph nodes, pulmonary consolidation and bone marrow (fig. 2e-h). Upper gastrointestinal endoscopic results were intact. A bone marrow smear showed that the rate of myelopoietic cells was increased but that of erythropoietic cells and megakaryocytes decreased, with normal cellularity. Neither tumor cells nor monoclonal cells were seen. The rate of eosinophils was not increased, and therefore, chronic myelocytic leukemia was not suspected (fig. 1c, d).

A needle biopsy of the cervical lymph node and endobronchial ultrasound-guided transbronchial needle aspiration were performed, and cytological examinations of the cervical lymph node and bronchial lavage fluid indicated adenocarcinoma. Immunohistochemical 
(IHC) examination showed cytokeratin $(\mathrm{CK}) 7(+), \mathrm{CK} 20(+), \operatorname{MUC1}(+), \operatorname{MUC2}(-), \mathrm{CEA}(+)$, p63(+), CD5(-/+), CDX2(-), human gastric mucin $(-/+)$, N-CAM(-), thrombomodulin(-), SPA(-), TTF-1(-), ALK(-), PSA(-), ER(-), PgR(-), HER2(-) and EGFR(-) expression (fig. 1e-h). The endobronchial ultrasound-guided transbronchial needle aspiration specimens were insufficient for diagnosis. Since the clinical and pathological investigations could not detect the primary site, the patient was diagnosed with GCSF-CUP.

After the approval of the intramural ethics committee and after having received the written informed consent from the patient, he was treated with carboplatin (AUC 6), paclitaxel $\left(175 \mathrm{mg} / \mathrm{m}^{2}\right)$, bevacizumab $(15 \mathrm{mg} / \mathrm{kg})$ and erlotinib $150 \mathrm{mg} /$ day. After the start of chemotherapy, G-CSF slightly decreased, but no change was seen on the CT scan (fig. 2i-l, fig. 3). Thirteen days later, intestinal perforation occurred. With the surgeon's consultation, the perforation was considered inoperable due to tumor invasion to the intestine. The patient was given palliative therapies and died 23 days after the start of chemotherapy. Treatment-related toxicity during chemotherapy was grade 4 anemia, grade 3 platelet count decrease, grade 1 aspartate aminotransferase elevation and grade 5 intestinal perforation.

His autopsy confirmed bulky lymph nodes of the neck, showing a remarkably shrunken appearance compared to before the therapy (fig. 1b), mediastinal and para-aortic lymphadenopathy and massive thickening of the mesentery. Perforation was observed in the ileocecal area with invasion of the tumor. Histopathological assessment proved poorly differentiated adenocarcinoma with no specific morphologic features, invasion to the right lung and intestine as well as lymph node metastasis, and a well-differentiated hepatocellular carcinoma without any metastasis or invasion in the liver. IHC examination of the mesentery showed the same results as those from the needle biopsy, and the tumor cells were positive for G-CSF with immunohistochemistry (fig. $2 \mathrm{~b}, \mathrm{e}-\mathrm{j}$ ).

\section{Discussion}

GCSF-PT causes remarkable peripheral leukocytosis and neutrophil production in the bone marrow. Although several reports have reviewed each site of GCSF-PT (e.g., G-CSFproducing gastric tumor), the frequency and histology are still unclear. We conducted a search in PubMed with the key words 'granulocyte colony-stimulating factor' or 'G-CSF', 'producing tumor' and 'case reports'; 176 cases are reported and 171 described in Japanese. Additionally, we explored the Japan Medical Abstracts Society database looking for the key words 'G-CSF', 'producing' and 'case report' in Japanese; 354 cases, including 102 cases from PubMed, have been described since June 2014. Hence, we reviewed 420 cases of GCSF-PT, with the exception of 4 cases that did not refer to the histopathology due to a lack of specimens.

GCSF-PT cases are classified according to the primary site and pathologically (table 2). The most common lesions of GCSF-PT are found in the lung, urinary tract (renal pelvis, ureter, urethra and bladder), stomach and duodenum, and the esophagus. Others included 2 malignant fibrous histiocytomas, 1 epithelioid sarcoma and 1 undifferentiated sarcoma in the soft tissue and bone; 1 squamous cell carcinoma in the middle ear; 1 squamous cell carcinoma in the head and neck; 1 squamous cell carcinoma of the urachus, and 1 prostate adenocarcinoma. GCSF-PT sometimes secretes other hormones simultaneously, such as parathyroid hormone-related protein $C$ in 21 cases, $\alpha$-fetoprotein in 3 cases , $\beta$-human chorionic gonadotropin in 2 cases, antidiuretic hormone in 1 case and erythropoietin in 1 case, respectively. Our case did not have any clinical feature of abnormal hormone secretion other than G-CSF. 
Yasui et al.: Granulocyte Colony-Stimulating Factor-Producing Carcinoma of Unknown Primary Site

Diagnostic criteria for GCSF-PT are the following: (1) extreme leukocytosis, (2) elevated G-CSF activity, (3) a decrease in the WBC count after tumor resection, or (4) proof of G-CSF production in the tumor [7]. Our case matched criteria 1,2 and 4 and was therefore diagnosed with GCSF-PT.

PET-CT scans show an abnormally high ${ }^{18} \mathrm{~F}-\mathrm{FDG}$ uptake, not only by the tumor itself but also diffusely throughout the bone marrow. This finding could be explained by the elevated bone marrow metabolism associated with the excessively active production of granulocytes under G-CSF stimulation and is considered to be useful for the diagnosis of GCSF-PT [8]. Moreover, we confirmed no malignancy with bone marrow puncture, and the increase in only myelopoietic cells is compatible with excessive G-CSF secretion.

Meanwhile, CUP is relatively rare, and standard clinical evaluation, unlike in most cancers, does not identify the anatomic primary site because of its extremely small size or possibly local regression due to antitumor immune defenses as well as its protracted clinical latency [9]. Since it is difficult to detect the primary organs, investigations with imaging, tumor and IHC markers are performed to at least classify the type of carcinoma for the purpose of a therapy guideline. However, $20 \%$ of CUP patients show clinical and/or pathologic features that fit into one or several defined treatable subsets (e.g., isolated axillary lymph node tumors of female patients are treated as stage II breast cancer). These patients have clinical features that strongly suggest a specific diagnosis, even though an anatomic primary site cannot be identified, and the prognosis is similar to these cancers. The remaining $80 \%$ of patients with CUP do not fall into any of these favorable subsets and have been treated with empiric chemotherapy [10]. In our case, CT, MRI and PET images as well as endoscopy did not detect the primary site. IHC examinations showed adenocarcinoma and CK7/CK20 positivity, generally suggesting transient cell carcinoma, pancreatic ductal carcinoma, cholangiocellular carcinoma and gastric adenocarcinoma. The results of MUC1, MUC2, CEA and CDX2 pointed to a possible pancreatic ductal carcinoma, and p63 positivity indicates ureteral carcinoma; however, thrombomodulin negativity suggests the opposite. A slight positivity of human gastric mucin implies gastric carcinoma, a negativity of N-CAM denies neurogenic tumor, and a CD5 result may show lymphocyte infiltration; other IHC stains are not helpful in predicting a likely primary tumor. According to these clinical and histopathological results, the primary site could not be detected; therefore, our case was diagnosed with CUP. The primary organ has been identified in $70 \%$ of CUP patients by autopsy, but our case has also failed in this matter [11]. So far, a combination of both GCSFPT and CUP does not seem to have been reported, and this study is likely the first case.

Although, because of the rarity of the disease, there is a lack of data from randomized phase III trials which would be helpful for the treatment of patients with CUP, the regimens with newer chemotherapeutic agents (e.g., taxanes, gemcitabine, irinotecan) show modest improvement; most of these newer regimens report response rates of $30-50 \%$, and the median survival time was 8-10 months. A phase II trial of the paclitaxel-carboplatin plus bevacizumab-erotinib regimen for CUP patients was reported in 2009. This regimen produced a response rate of 53\%; the median overall survival time was 12.6 months, the median progression-free survival 8 months, and the 1- and 2-year survival rates 51 and $27 \%$, respectively. Both the median progression-free survival and 2-year survival rate are the best among previously reported empiric chemotherapy regimens [5]. Similar to the reported effectiveness, this new regimen was also presumed to be efficacious in our case because a decrease in G-CSF and WBC was observed after the treatment and because the appearance of the bulky neck lymph node remarkably shrank. 


\begin{tabular}{l|l}
\hline DOI: $10.1159 / 000369335$ & $\begin{array}{l}\text { C 2014 S. Karger AG, Basel } \\
\text { www.karger.com/cro }\end{array}$ \\
\hline
\end{tabular}

Yasui et al.: Granulocyte Colony-Stimulating Factor-Producing Carcinoma of Unknown Primary Site

\section{Conclusions}

We herein report a case of GCSF-CUP. Simultaneous CUP and GCSF-PT is rare and has a poor prognosis. If leukocytosis is detected in relation to a nonlymphoid hematopoietic malignant tumor, GCSF-PT should be reckoned. Careful observation is paramount in CUP treatment due to the unpredictable nature of its metastatic potential and pattern.

\section{Acknowledgement}

This work was supported by the Central Japan Lung Study Group (CJLSG).

\section{Disclosure Statement}

The authors declare no conflicts of interest.

\section{References}

1 Robinson WA: Granulocytosis in neoplasia. Ann NY Acad Sci 1974;230:212-218.

2 Lieschke GJ, Burgess AW: Granulocyte colony-stimulating factor and granulocyte-macrophage colonystimulating factor (1). N Engl J Med 1992;327:28-35.

-3 Lieschke GJ, Burgess AW: Granulocyte colony-stimulating factor and granulocyte-macrophage colonystimulating factor (2). N Engl J Med 1992;327:99-106.

4 Savarese TM, Mitchell K, McQuain C, et al: Coexpression of granulocyte colony stimulating factor and its receptor in primary ovarian carcinomas. Cancer Lett 2001;162:105-115.

5 Morris GJ, Greco FA, Hainsworth JD, et al: Cancer of unknown primary site. Semin Oncol 2010;37:71-79.

6 Hirotoshi Y, Kazuhide S, Yoshihiro T, et al: Carcinoma of unknown primary site treated with carboplatin + paclitaxel + bevacizumab + erlotinib and its maintenance chemotherapy. Case Rep Oncol 2014;7:583-590.

7 Asano S, Urabe A, Okabe T, et al: Demonstration of granulopoietic factor(s) in the plasma of nude mice transplanted with a human lung cancer and in the tumor tissue. Blood 1977;49:845-852.

8 Miyako M, Kazuo K, Yuji M, et al: 18F-FDG-PET/CT findings of granulocyte colony stimulating factor (G-CSF)producing lung tumors. Ann Nucl Med 2008;22:635-639.

-9 Alberti C: Carcinoma of unknown primary (CUP); some considerations about pathogenesis and diagnostic strategy, particularly focusing on CUPS pertaining to the Urology. G Chir 2012;33:41-46.

10 Hainsworth JD, Greco FA: Gene expression profiling in patients with carcinoma of unknown primary site: from translational research to standard of case. Virchows Arch 2014;464:393-402.

11 Pentheroudakis G, Golfinopoulous V, Pavilidis N: Switching benchmarks in cancer of unknown primary: from autopsy to microarray. Eur J Cancer 2007;43:2026-2036. 


DOI: $10.1159 / 000369335$

(C) 2014 S. Karger AG, Base www.karger.com/cro

Yasui et al.: Granulocyte Colony-Stimulating Factor-Producing Carcinoma of Unknown Primary Site

Table 1. Blood biochemistry and tumor markers at first arrival and a few weeks after therapy start

\begin{tabular}{|c|c|c|c|c|c|}
\hline WBC & $25,120 / \mu \mathrm{l}$ & $\mathrm{TP}$ & $7.2 \mathrm{~g} / \mathrm{dl}$ & CEA & $5.1 \mathrm{ng} / \mathrm{ml}$ \\
\hline Neutrophils & $82.3 \%$ & AST & 24 units/l & SLX & 58.6 units $/ \mathrm{ml}$ \\
\hline Lymphocytes & $8.2 \%$ & ALT & 12 units/l & SCC & $114.6 \mathrm{ng} / \mathrm{ml}$ \\
\hline Monocytes & $2.9 \%$ & LDH & 515 units/l & CYFRA & $500.2 \mathrm{ng} / \mathrm{ml}$ \\
\hline Eosinophils & $6.3 \%$ & ALP & 371 units/l & ProGRP & $58.7 \mathrm{pg} / \mathrm{ml}$ \\
\hline \multirow[t]{2}{*}{ Basophils } & $0.3 \%$ & $\gamma \mathrm{GTP}$ & 41 units/l & NSE & $47.6 \mathrm{ng} / \mathrm{ml}$ \\
\hline & & T-Bil & $0.6 \mathrm{mg} / \mathrm{dl}$ & CA125 & 73.8 units $/ \mathrm{ml}$ \\
\hline $\mathrm{RBC}$ & $431 \times 10^{4} / \mu \mathrm{l}$ & BUN & $13 \mathrm{mg} / \mathrm{dl}$ & CA602 & 37.2 units $/ \mathrm{ml}$ \\
\hline $\mathrm{Hb}$ & $13.4 \mathrm{~g} / \mathrm{dl}$ & Cre & $0.68 \mathrm{mg} / \mathrm{dl}$ & CA15-3 & $62.3 \mathrm{ng} / \mathrm{ml}$ \\
\hline $\mathrm{Ht}$ & $40.1 \%$ & $\mathrm{Na}$ & $137 \mathrm{mEq} / \mathrm{l}$ & BCA225 & 82 units $/ \mathrm{ml}$ \\
\hline \multirow[t]{4}{*}{ Plt } & $21.8 \times 10^{4} / \mu \mathrm{l}$ & $\mathrm{K}$ & $3.6 \mathrm{mEq} / \mathrm{l}$ & CA19-9 & 238.7 units $/ \mathrm{ml}$ \\
\hline & & $\mathrm{Cl}$ & $101 \mathrm{mEq} / \mathrm{l}$ & PSA & $1.273 \mathrm{ng} / \mathrm{ml}$ \\
\hline & & CRP & $4.46 \mathrm{mg} / \mathrm{dl}$ & sIL2-R & 1,040 units $/ \mathrm{ml}$ \\
\hline & & & & AFP & 3.8 units/ml \\
\hline
\end{tabular}

The examinations showed leukocytosis, elevated CRP and some elevated tumor markers. RBC = Red blood cells; $\mathrm{Hb}=$ hemoglobin; $\mathrm{Ht}$ = hematocrit; Plt = platelets. 


\section{Case Reports in Oncology}

\begin{tabular}{l|l}
\hline Case Rep Oncol 2014;7:780-788 \\
\hline DOI: $10.1159 / 000369335$ & $\begin{array}{l}\text { C 2014 S. Karger AG, Basel } \\
\text { www.karger.com/cro }\end{array}$ \\
\hline
\end{tabular}

Yasui et al.: Granulocyte Colony-Stimulating Factor-Producing Carcinoma of Unknown Primary Site

Table 2. G-CSF-producing cases (primary site and histology with the number of cases)

\begin{tabular}{|c|c|c|c|}
\hline Lung & 94 & Thyroid & 14 \\
\hline Large cell carcinoma & 34 & Undifferentiated carcinoma & 12 \\
\hline Carcinoma with pleomorphic/sarcomatoid/sarcomatous elements & 25 & Adenosquamous cell carcinoma & 1 \\
\hline Adenocarcinoma & 22 & Papillary carcinoma & 1 \\
\hline Squamous cell carcinoma & 10 & Kidney & 13 \\
\hline Malignant fibrous histiocytoma & 3 & Renal cell carcinoma & 8 \\
\hline Urinary tract & 57 & Collecting duct carcinoma & 3 \\
\hline Transitional cell carcinoma & 40 & Metanephric adenoma & 1 \\
\hline Squamous cell carcinoma & 11 & Transitional cell carcinoma & 1 \\
\hline Undifferentiated carcinoma & 3 & Ovary & 12 \\
\hline Sarcomatoid carcinoma & 2 & Undifferentiated & 4 \\
\hline Giant cell carcinoma & 1 & Mucinous tumors & 2 \\
\hline Stomach, duodenum & 38 & Serous tumors & 1 \\
\hline Adenocarcinoma & 27 & Endometrioid adenocarcinoma & 1 \\
\hline Adenosquamous cell carcinoma & 6 & Cystoadenocarcinofibroma & 1 \\
\hline Squamous cell carcinoma & 2 & Adenosarcoma & 1 \\
\hline Undifferentiated carcinoma & 1 & Transitional cell carcinoma & 1 \\
\hline Mucosa-associated lymphoid tissue lymphoma & 1 & Squamous cell carcinoma & 1 \\
\hline Malignant fibrous histiocytoma & 1 & Pleura & 10 \\
\hline Esophagus & 30 & Malignant mesothelioma & 10 \\
\hline Squamous cell carcinoma & 14 & Mouth, tongue, jaw & 10 \\
\hline Carcinosarcoma & 14 & Squamous cell carcinoma & 9 \\
\hline Adenosquamous cell carcinoma & 1 & Spindle cell carcinoma & 1 \\
\hline Undifferentiated & 1 & Skin & 8 \\
\hline Liver & 23 & Squamous cell carcinoma & 6 \\
\hline Hepatocellular carcinoma & 11 & Malignant melanoma & 1 \\
\hline Intrahepatic bile duct carcinoma & 5 & Angiosarcoma & 1 \\
\hline Adenosquamous cell carcinoma & 2 & Thymus, mediastinum & 7 \\
\hline Sarcomatoid carcinoma & 1 & Thymic carcinoma & 4 \\
\hline Leiomyosarcoma & 1 & Teratoma & 2 \\
\hline Undifferentiated carcinoma & 1 & Undifferentiated carcinoma & 1 \\
\hline Unclassified tumor & 1 & Pharynx, larynx, tonsil & 7 \\
\hline Pancreas & 20 & Squamous cell carcinoma & 5 \\
\hline Anaplastic carcinoma & 9 & Spindle cell carcinoma & 2 \\
\hline Adenocarcinoma & 8 & Salivary gland & 6 \\
\hline Adenosquamous cell carcinoma & 3 & Adenocarcinoma & 2 \\
\hline Uterus & 20 & Squamous cell carcinoma & 2 \\
\hline Squamous cell carcinoma & 12 & Undifferentiated carcinoma & 1 \\
\hline Adenocarcinoma & 3 & Mucoepidermoid carcinoma & 1 \\
\hline Sarcoma & 3 & Sinus, nasal cavity & 4 \\
\hline Small cell carcinoma & 1 & Squamous cell carcinoma & 4 \\
\hline Endometrioid adenocarcinoma & 1 & Breast & 4 \\
\hline Biliary tract, gallbladder & 16 & Squamous cell carcinoma & 2 \\
\hline Adenocarcinoma & 9 & Invasive ductal carcinoma & 1 \\
\hline Adenosquamous cell carcinoma & 4 & Phyllodes tumor & 1 \\
\hline Squamous cell carcinoma & 2 & Peritoneum, mesentery & 4 \\
\hline Sarcomatoid carcinoma & 2 & Adenocarcinoma & 2 \\
\hline Intestine & 15 & Malignant mesothelioma & 1 \\
\hline Undifferentiated carcinoma & 5 & Liposarcoma & 1 \\
\hline Adenocarcinoma & 4 & Others & 8 \\
\hline Malignant fibrous histiocytoma & 3 & & \\
\hline Leiomyosarcoma & 1 & & \\
\hline
\end{tabular}




\section{Case Reports in Oncology}
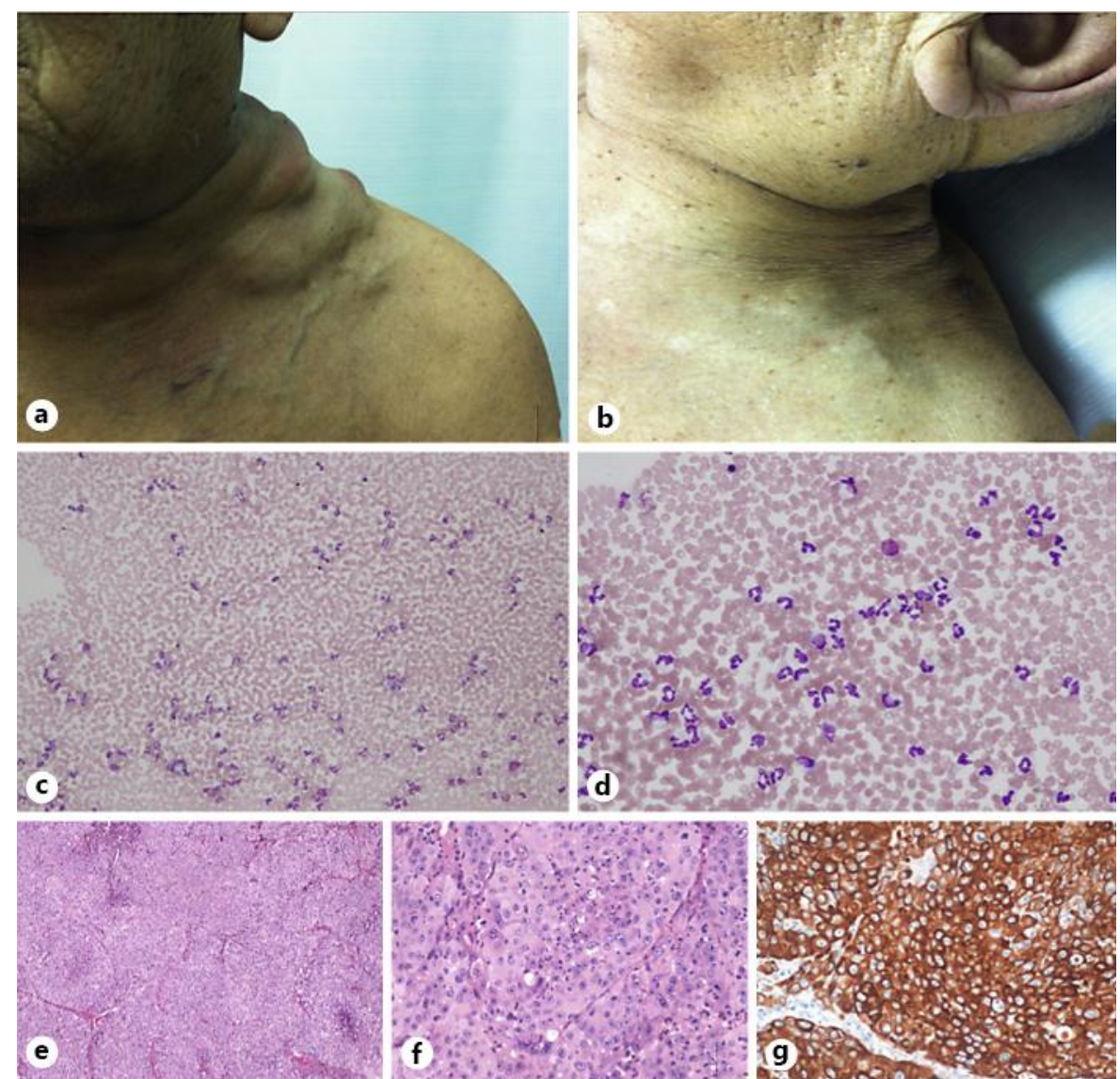

Yasui et al.: Granulocyte Colony-Stimulating Factor-Producing Carcinoma of Unknown Primary Site

(C) 2014 S. Karger AG, Basel www.karger.com/cro
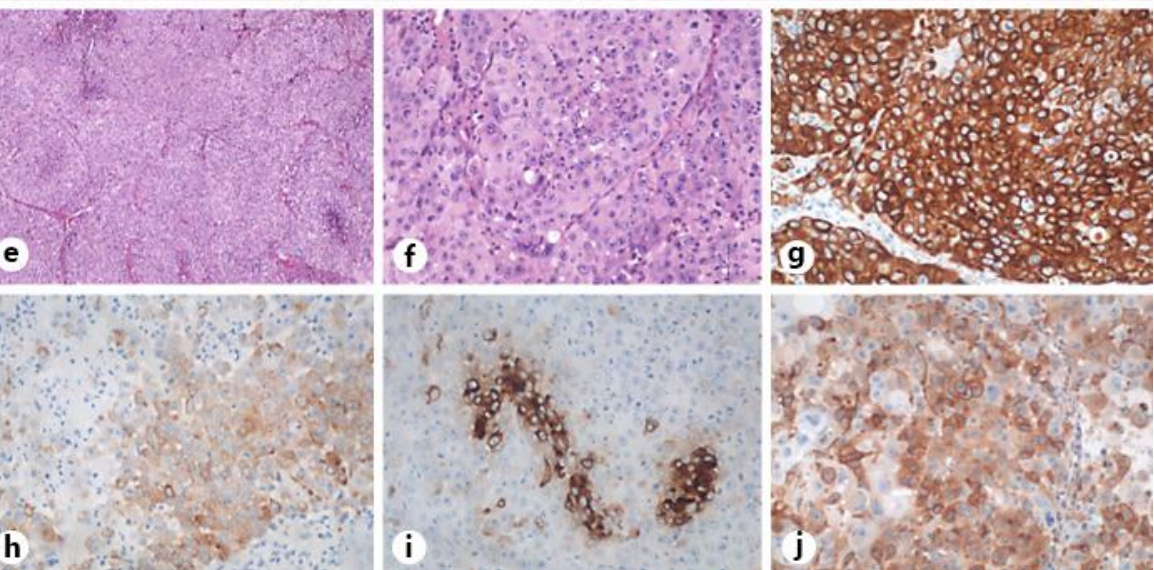

Fig. 1. a The patient's neck showing swollen lymph nodes at the first visit. b Cervical lymph nodes appeared to be shrunken at the autopsy. c, d Bone marrow smear. Romanowsky stain. $\times 100$ (c); $\times 400$ (d). e, $f$ Metastatic adenocarcinoma of unknown primary origin in the peritoneum discovered at autopsy; histopathologic examinations. Hematoxylin and eosin stain. $\times 40$ (e); $\times 400$ (f). g CK7 stain. h CK20 stain (positive). i CEA stain (positive). j Anti-G-CSF antibody (positive). 


\section{Case Reports in Oncology}
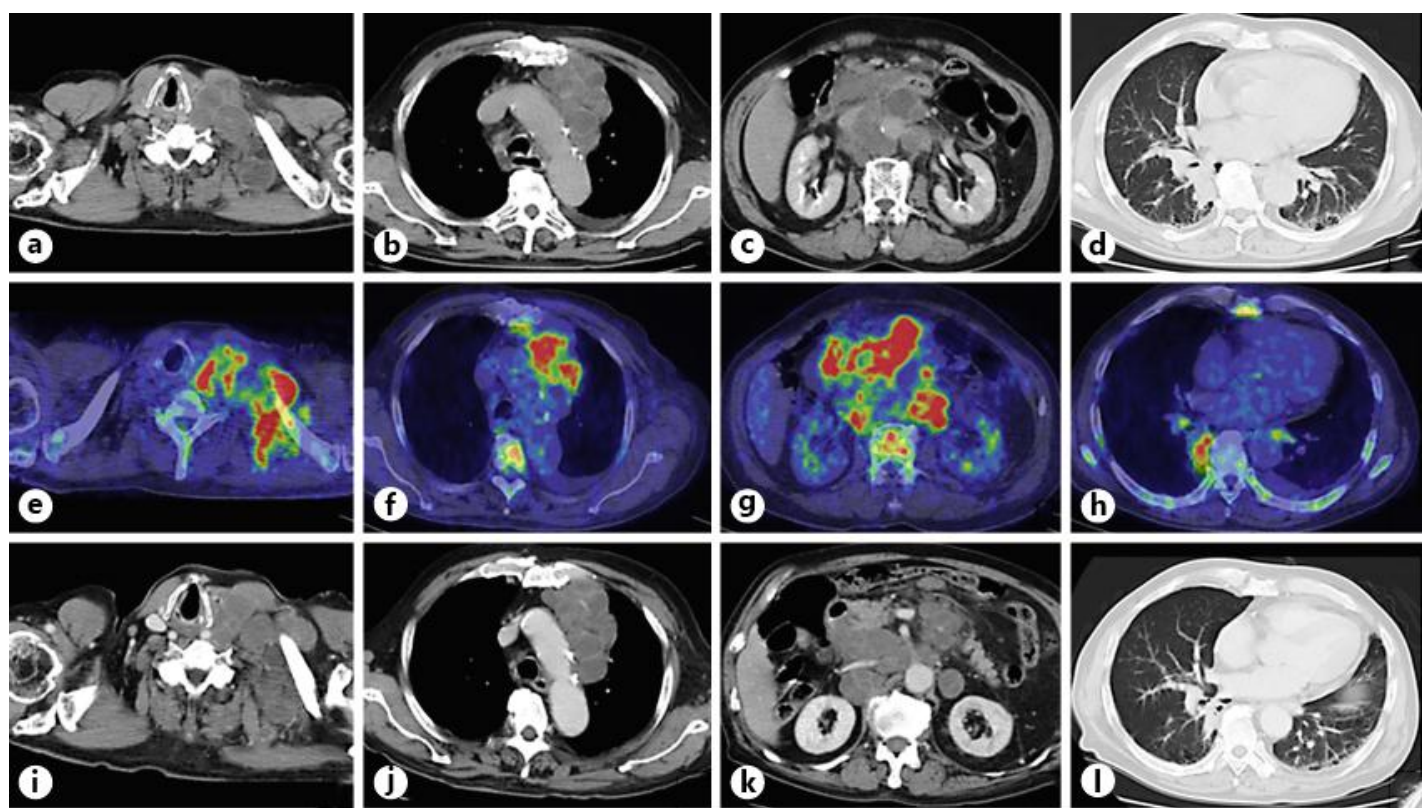

Fig. 2. a-c Bulky lymph nodes of the left cervix, mediastinum and peritoneum. $\mathbf{d}$ Consolidation in the right lower lung. $\mathbf{e}-\mathbf{h}$ FDG accumulation in the metastatic lesions of the cervical lymph nodes, mediastinum, peritoneum and consolidation of the lung; it also shows accumulation in the bone marrow. i-I Lymph node metastases and lung consolidation did not change.

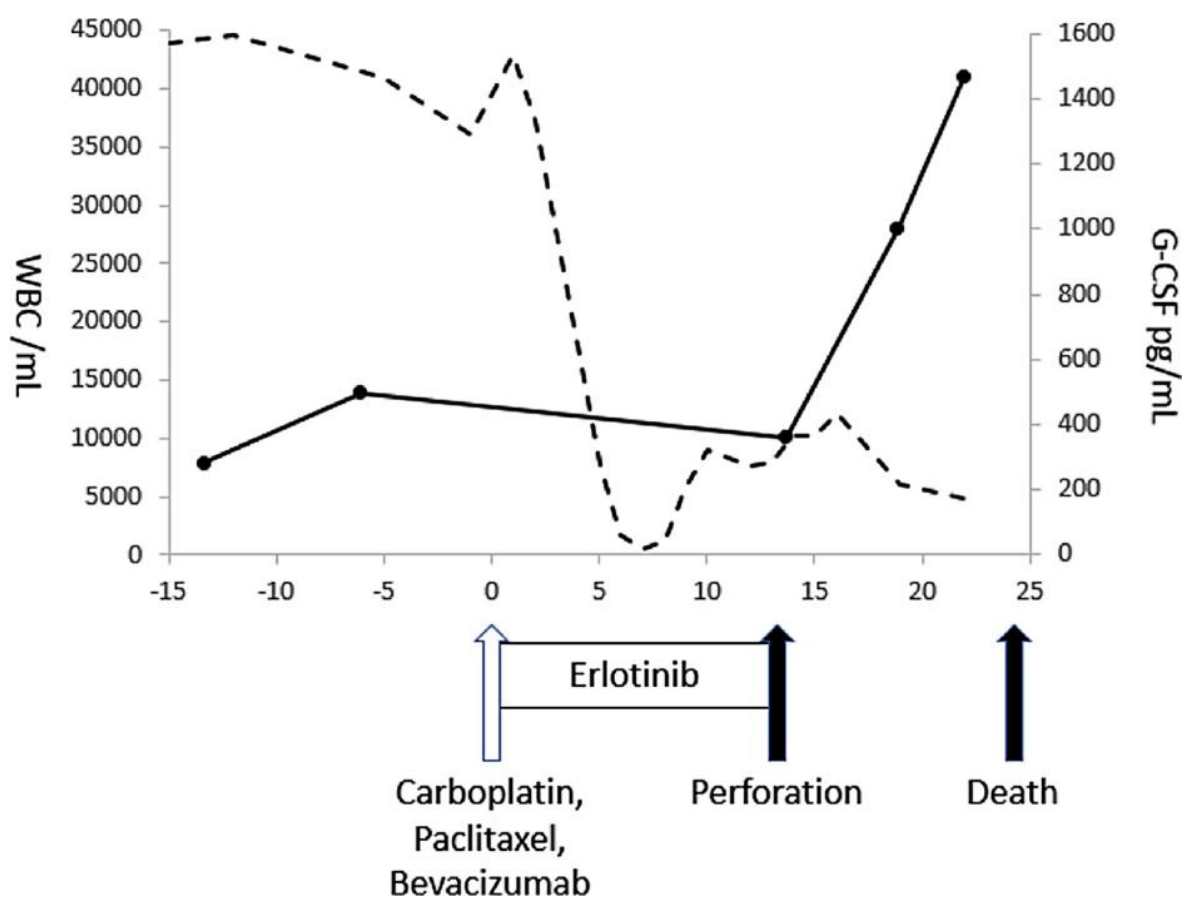

Fig. 3. Overview of the therapy and changes in WBC and G-CSF. The arrows indicate the chemotherapies (CBDCA, PTX and BEV). The solid line shows the G-CSF and the dashed line the WBC count, respectively. 